\title{
Adjuvant EGFR tyrosine kinase inhibitors in EGFR-mutant non-small cell lung cancer: still an investigational approach
}

\author{
Jacek Jassem \\ Department of Oncology and Radiotherapy, Medical University of Gdańsk, Gdańsk, Poland \\ Correspondence to: Jacek Jassem. Department of Oncology and Radiotherapy, Medical University of Gdańsk, 7 Dębinki St. 80-211, Gdańsk, Poland. \\ Email: jjassem@gumed.edu.pl. \\ Provenance: This is an invited article commissioned by the Academic Editor Dr. Zhizhou Yang (Washington University School of Medicine, St. Louis, \\ MO, USA). \\ Comment on: Pennell NA, Neal JW, Chaft JE, et al. SELECT: A Phase II Trial of Adjuvant Erlotinib in Patients With Resected Epidermal Growth \\ Factor Receptor-Mutant Non-Small-Cell Lung Cancer. J Clin Oncol 2019;37:97-104.
}

Submitted Aug 18, 2019. Accepted for publication Aug 30, 2019.

doi: $10.21037 /$ tlcr.2019.09.02

View this article at: http://dx.doi.org/10.21037/tlcr.2019.09.02

The development of epidermal growth factor receptor tyrosine kinase inhibitors (EGFR-TKIs) have revolutionized treatment paradigms in lung cancer. Currently, EGFR TKIs constitute first- and second-line treatment of choice for EGFR-mutant advanced non-small cell lung cancer (NSCLC) patients. The first-generation TKIs (gefitinib, erlotinib and icotinib) have been supplemented by second (afatinib and dacomitinib) and third-generation (osimertinib) molecules. Several other EGFR-TKIs are currently being investigated in clinical trials (1).

Typically, targeted anti-cancer therapies in solid malignancies are first investigated in advanced stages, and subsequently agents with confirmed activity are promptly submitted to studies in early disease. An example of such development includes trastuzumab, a monoclonal antibody used in human type 2 EGFR (HER2) positive breast cancer. The first phase 3 study reporting the high efficacy of this agent in metastatic disease was published in 2001 (2), and just 4 years later three large randomized trials demonstrated its value in the adjuvant setting $(3,4)$. Adjuvant studies using trastuzumab in breast cancer included an impressive total number of around 20,000 patients, and resulted in widespread implementation of this compound in the preoperative and postoperative settings. Another spectacular example is imatinib, a TKI used in gastrointestinal stromal tumor, a relatively rare malignancy. Efficacy of this compound in an adjuvant setting (5) was documented 7 years after the first report showing its astonishing activity in advanced disease (6). Just 3 more years were needed to establish the optimal 3-year duration of adjuvant treatment (7). Similarly, only 3 years elapsed from the first trial showing the efficacy of dabrafenib plus trametinib, drugs that target the mitogen-activated protein kinase (MAPK) pathway in advanced malignant melanoma with BRAF V600 mutations (8), to the demonstration of their benefit in adjuvant treatment (9).

At present, the only standard systemic adjuvant treatment option in operable stage II-III NSCLC, regardless of EGFR mutation status, is cytotoxic chemotherapy, despite its mere 5 -year overall survival (OS) gain of 5\% (10).

Until now only five randomized studies have been performed to assess EGFR-TKIs in operable NSCLC (Table 1). Two of these studies compared EGFR TKI $v$ s. placebo $(11,12)$, one chemotherapy followed by EGFR TKI vs. chemotherapy alone (13), and two EGFR TKI $v s$. chemotherapy $(14,15)$. The results of two early studies including molecularly unselected patients were negative. The prematurely closed NCIC CTG BR19 study did not show disease-free survival (DFS) or OS benefit of gefitinib for 2 years compared to placebo (11). Similarly, no superiority was found from adjuvant erlotinib $v s$. placebo in the RADIANT study (12), which included patients with EGFR protein-positive tumors by immunohistochemistry or with $E G F R$ amplification by fluorescence in situ, the biomarkers currently considered ineffective in selection for EGFR TKIs. 
Table 1 Completed randomized studies of postoperative therapy with EGFR TKIs in NSCLC

\begin{tabular}{|c|c|c|c|c|c|c|c|c|c|}
\hline Study & Phase & $\begin{array}{l}\text { Selection } \\
\text { criteria }\end{array}$ & Stage & Region & $\begin{array}{c}\mathrm{N} \\
\text { (total) }\end{array}$ & $\begin{array}{c}\mathrm{N} \\
(\mathrm{m} E G F R)\end{array}$ & Study arms & $\begin{array}{l}\text { Primary } \\
\text { endpoint }\end{array}$ & Main results (mEGFR) \\
\hline BR19 (11) & III & All NSCLC & IBIIIA & $\begin{array}{c}\text { North } \\
\text { America }\end{array}$ & 503 & 15 & G vs. placebo (2 y) & OS & NR \\
\hline RADIANT (12) & III & $\begin{array}{l}\text { EGFR+ by } \\
\text { IHC or FISH }\end{array}$ & IBIIIA & Global & 973 & 161 & E vs. placebo (2 y) & OS & $\begin{array}{l}\text { OS NR; median DFS } \\
46.4 \text { vs. } 28.5 \text { m (NS) }\end{array}$ \\
\hline EVAN (14) & II & mEGFR & IIIA & China & 102 & 102 & $\mathrm{E}(2 \mathrm{y})$ vs. $4 \times \mathrm{PV}$ & DFS & $\begin{array}{c}\text { Two-year DFS rate } 81 \% \\
\text { vs. } 54 \%(P=0.01)\end{array}$ \\
\hline ADJUVANT (15) & III & mEGFR & $\begin{array}{c}I I-I I I A \\
(\mathrm{~N} 1-\mathrm{N} 2)\end{array}$ & China & 222 & 222 & $\mathrm{G}(2 \mathrm{y})$ vs. $4 \times \mathrm{PV}$ & DFS & $\begin{array}{c}\text { Median DFS } 28.7 \text { vs. } \\
18.0 \mathrm{~m}(\mathrm{P}=0.005)\end{array}$ \\
\hline
\end{tabular}

EGFR TKI, epidermal growth factor receptor tyrosine kinase inhibitor; NSCLC, non-small cell lung cancer; mEGFR, patients with EGFR mutation; G, gefitinib; OS, overall survival; NR, not reported; IHC, immunochemistry; FISH, fluorescence in situ hybridization; E, erlotinib; DFS, disease free survival; NS, not significant; PC, pemetrexed plus cisplatin; PV, cisplatin plus vinorelbine.

As expected, more promising were the results of three completed studies (all performed in China), enrolling selected patients with EGFR-mutant tumors. The first of them, a small phase 2 trial, showed significantly longer DFS of gefitinib for 6 months following pemetrexedcarboplatin combination, compared with chemotherapy alone (13). Another randomized phase 2 study (EVAN) showed significantly higher 2-year DFS of erlotinib for 2 years compared to four cycles of cisplatin-vinorelbine chemotherapy (14). The only phase 3 study (ADJUVANT) showed significantly longer DFS of gefitinib for 2 years compared to four cycles of a cisplatin-vinorelbine combination, but general results were disappointingly grim, with almost all patients relapsing within 4 years (15). The meta-analysis of the five above-mentioned randomized studies (including only patients with EGFR mutation) showed increased DFS with EGFR-TKI-based regimens (HR 0.52; 95\% CI: 0.34-0.78, $\mathrm{P}=0.002$ ), but this was not translated into OS benefit (16).

Most recently presented were the results of an open-label single-arm phase 2 study (SELECT) performed in the USA, that investigated the efficacy of adjuvant erlotinib in patients with EGFR-mutant early-stage NSCLC (17). Considering the scarcity of data on adjuvant EGFR TKIs in the nonAsian populations, this study has raised great interest. The SELECT study included 100 stage IA-IIIA patients and was powered to demonstrate a 2 -year DFS greater than $86 \%$, i.e., $10 \%$ more compared with the historic figure of $76 \%$. After a median follow-up of 5.2 years, this primary endpoint was met: 2-year DFS was $88 \%$. The median DFS and OS was not reached, the 5-year DFS was $56 \%$ (95\% CI: $45-$ $66 \%$ ), and the 5 -year OS was $86 \%$ (95\% CI: 77-92\%). Disease recurred in 40 patients, including four while on adjuvant erlotinib. Treatment adherence was relatively poor, with $40 \%$ of patients requiring dose reductions by $50 \%$, and $16 \%$ further reduction to $25 \%$ of the original dose. Of the 36 patients with recurrence after completing erlotinib treatment, 26 were retreated with this compound, and the majority of them derived clinical benefit from re-exposure. Interestingly, of the 20 patients who had determined $E G F R$ mutation status of the recurrent tumor, all but one maintained the original canonical EGFR mutation pattern. The only patient with acquired $T 790 \mathrm{M}$ resistance mutation was among those four who developed progression while receiving adjuvant erlotinib. This may imply the hypothesis that EGFR TKIs inhibit rather than kill cancer cells, and that prolonged anti-EGFR treatment is unlikely to induce resistance mechanisms. The SELECT study was properly designed and executed and provides another signal for potential role of EGFR TKI in adjuvant setting. However, due to all the limitations of single-arm design, it still does not provide strong evidence.

In view of the relatively high incidence of $E G F R$-mutant NSCLC, the number of studies investigating the role of EGFR TKI in an adjuvant setting, and the total number of participating patients is strikingly low. It is really difficult to explain the reluctance to carry out more trials addressing this concept. Additionally, the quality of randomized studies performed so far has been relatively low in terms of patient selection, study design and execution. All three randomized 
studies enrolling exclusively $E G F R$-mutated patients were small and included the Chinese population. Patients from East Asia comprise a higher proportion of EGFR-mutant cases than those from other geographical regions, and have distinctive clinicopathologic features. Hence, there is the question of whether the results of these studies may be generalized, even though $83 \%$ of the SELECT study subjects were of non-Asian ethnicity.

Importantly, none of the completed studies in patients selected by EGFR mutation used OS as the primary endpoint. In consequence there are no robust data on OS impact of adjuvant EGFR TKIs. Further, the available results cannot indicate whether chemotherapy should be replaced or supplemented by an EGFR-TKI. Using EGFR TKIs alone may be viewed as more appealing, as it avoids the burden of chemotherapy toxicity. On the other hand, the combined approach may be potentially more efficient in view of the potential NSCLC heterogeneity. Namely, it may be speculated, that tumors containing both EGFRmutated and $E G F R$ wild-type clones may derive benefit from complementary mechanisms of action.

An important and unresolved question remains the duration of EGFR TKI treatment. Of the five completed studies, four employed a 2-year therapy, but this may be considered a purely empiric approach. Indeed, in advanced NSCLC most responses to EGFR TKIs occur within the first 2-3 months of treatment. This puts in doubt the validity of prolonged treatment, given its toxicity and cost. Although targeted therapy is generally considered less toxic and better tolerated than cytotoxic chemotherapy, it carries prolonged and troublesome skin and gastrointestinal side effects. Two-year EGFR TKI treatment instead of 3 months of chemotherapy may be burdensome and raises the question of patient adherence. Actually, treatment compliance in clinical studies was relatively low, and up to one-third of patients could not receive a 2-year medication. EGFR TKIs therapy is also much more costly, and in some insurance systems may create substantial financial problems for patients. An ongoing phase II trial (NCT01746251) compares 3 months vs. 2 years of postoperative therapy with afatinib in $E G F R$-mutated NSCLC.

All completed studies to date used first-generation EGFR TKIs which bind reversibly to EGFR harboring sensitizing mutations (mostly exon 19 deletions and exon 21 substitution), but also to wild-type EGFR, thus increasing treatment toxicity. Additionally, these agents demonstrate marginal inhibition of exon 20 T790M mutant EGFR, constituting a common resistance mechanism. Osimertinib, a third-generation EGFR TKI, has a minimal inhibition of wild-type EGFR, resulting in lower toxicity (18). This compound is also more potent, and has a strong affinity for sensitizing and resistance T790M mutation (19).

The role of EGFR TKIs in an adjuvant setting is the subject of a few ongoing clinical studies. In the ALCHEMIST-EGFR (NCT02193282) trial, initiated in 2014, EGFR-mutant NSCLC patients are randomized to 2-year erlotinib or placebo, both preceded by adjuvant chemotherapy. Notably, this trial is being run in parallel with a similar study (ALCHEMIST-ALK, E4512), comparing anaplastic lymphoma kinase (ALK) inhibitor crizotinib vs. placebo in NSCLC patients with identified $A L K$ gene rearrangement. In the WJOG6410L phase III trial, initiated in Japan in 2012, stage II-III NSCLC patients harboring EGFR mutations are randomly assigned to gefitinib for 2 years, or four cycles of cisplatin-vinorelbine combination. In a similar study (NCT02448797), carried out in China since 2015 , patients are randomized to cisplatin in combination with vinorelbine or pemetrexed, or 2-year icotinib. Another Chinese phase III study (NCT01996098), initiated in 2013, compares chemotherapy $v s$. icotinib administered for 6 or 12 months. In the international ADAURA trial (NCT02511106) patients are assigned to 3 -year osimertinib treatment or placebo. Out of the running studies, only one (ALCHEMIST) uses OS as the primary endpoint, whereas all the others employ DFS.

Do the currently available data justify considering EGFR TKIs as a new paradigm of adjuvant therapy for EGFRmutant NSCLC patients? On one hand, the results seem encouraging, but the evidence is relatively weak and many questions remain unresolved. Hence, the use of adjuvant EGFR TKIs should still be considered an investigational approach. Ongoing studies can add to the current knowledge and may change the standards of adjuvant treatment in oncogene-addicted early stage NSCLC.

\section{Acknowledgments}

None.

\section{Footnote}

Conflicts of Interest: The author declares following potential conflicts of interest: Speaker: AstraZeneca, Roche, Pfizer; Advisory roles: AstraZeneca, BMS, Pfizer, MSD, Takeda; 
Travel support: Roche, Pfizer.

Ethical Statement: The author is accountable for all aspects of the work in ensuring that questions related to the accuracy or integrity of any part of the work are appropriately investigated and resolved.

\section{References}

1. Tan CS, Kumarakulasinghe NB, Huang YQ, et al. Third generation EGFR TKIs: current data and future directions. Mol Cancer 2018;17:29.

2. Slamon DJ, Leyland-Jones B, Shak S, et al. Use of chemotherapy plus a monoclonal antibody against HER2 for metastatic breast cancer that overexpresses HER2. N Engl J Med 2001;344:783-92.

3. Piccart-Gebhart MJ, Procter M, Leyland-Jones B, et al. Trastuzumab after adjuvant chemotherapy in HER2positive breast cancer. N Engl J Med 2005;353:1659-72.

4. Romond EH, Perez EA, Bryant J, et al. Trastuzumab plus adjuvant chemotherapy for operable HER2-positive breast cancer. N Engl J Med 2005;353:1673-84.

5. Dematteo RP, Ballman KV, Antonescu CR, et al. Adjuvant imatinib mesylate after resection of localised, primary gastrointestinal stromal tumour: a randomised, doubleblind, placebo-controlled trial. Lancet 2009;373:1097-104.

6. Demetri GD, von Mehren M, Blanke CD, et al. Efficacy and safety of imatinib mesylate in advanced gastrointestinal stromal tumors. N Engl J Med 2002;347:472-80.

7. Joensuu H, Eriksson M, Sundby Hall K, et al. One vs three years of adjuvant imatinib for operable gastrointestinal stromal tumor: a randomized trial. JAMA 2012;307:1265-72.

8. Long GV, Stroyakovskiy D, Gogas H, et al. Combined BRAF and MEK inhibition versus BRAF inhibition alone in melanoma. N Engl J Med 2014;371:1877-88.

9. Long GV, Hauschild A, Santinami M, et al. Adjuvant Dabrafenib plus Trametinib in Stage III BRAF-Mutated Melanoma. N Engl J Med 2017;377:1813-23.

10. Arriagada R, Bergman B, Dunant A, et al. Cisplatinbased adjuvant chemotherapy in patients with completely resected non-small-cell lung cancer. N Engl J Med

Cite this article as: Jassem J. Adjuvant EGFR tyrosine kinase inhibitors in EGFR-mutant non-small cell lung cancer: still an investigational approach. Transl Lung Cancer Res 2019;8(Suppl 4):S387-S390. doi: 10.21037/tlcr.2019.09.02
2004;350:351-60.

11. Goss GD, O'Callaghan C, Lorimer I, et al. Gefitinib versus placebo in completely resected non-small-cell lung cancer: results of the NCIC CTG BR19 study. J Clin Oncol 2013;31:3320-6.

12. Kelly K, Altorki NK, Eberhardt WE, et al. Adjuvant Erlotinib Versus Placebo in Patients With Stage IBIIIA Non-Small-Cell Lung Cancer (RADIANT): A Randomized, Double-Blind, Phase III Trial. J Clin Oncol 2015;33:4007-14.

13. Li N, Ou W, Ye X, et al. Pemetrexed-carboplatin adjuvant chemotherapy with or without gefitinib in resected stage IIIA-N2 non-small cell lung cancer harbouring EGFR mutations: a randomized, phase II study. Ann Surg Oncol 2014;21:2091-6.

14. Yue D, Xu S, Wang Q, et al. Erlotinib versus vinorelbine plus cisplatin as adjuvant therapy in Chinese patients with stage IIIA EGFR mutation-positive non-small-cell lung cancer (EVAN): a randomised, open-label, phase 2 trial. Lancet Respir Med 2018;6:863-73.

15. Zhong WZ, Wang Q, Mao WM, et al. Gefitinib versus vinorelbine plus cisplatin as adjuvant treatment for stage II-IIIA (N1-N2) EGFR-mutant NSCLC (ADJUVANT/ CTONG1104): a randomised, open-label, phase 3 study. Lancet Oncol 2018;19:139-48.

16. Wu JX, He Q, Ye F, et al. EGFR-TKI-based vs nonEGFR-TKI-based adjuvant therapy in resected non-smallcell lung cancer with EGFR mutations: a meta-analysis of randomized controlled trials. Onco Targets Ther 2018;11:6803-10.

17. Pennell NA, Neal JW, Chaft JE, et al. SELECT: A Phase II Trial of Adjuvant Erlotinib in Patients With Resected Epidermal Growth Factor Receptor-Mutant Non-SmallCell Lung Cancer. J Clin Oncol 2019;37:97-104.

18. Cross DA, Ashton SE, Ghiorghiu S, et al. AZD9291, an irreversible EGFR TKI, overcomes T790M-mediated resistance to EGFR inhibitors in lung cancer. Cancer Discov 2014;4:1046-61.

19. Soria JC, Ohe Y, Vansteenkiste J, et al. Osimertinib in Untreated EGFR-Mutated Advanced Non-Small-Cell Lung Cancer. N Engl J Med 2018;378:113-25. 